\title{
Sappanone A inhibits oxidative stress, inflammation and apoptosis in cigarette smoke-induced human bronchial epithelial cells through regulating $\mathrm{Nrf2/HO-1}$ and TLR4/NF-kB signaling pathways
}

\author{
Yan Zhang \\ Huaihe Hospital of Henan University \\ Shanshan Wang \\ Huaihe Hospital of Henan University \\ Hongli Li \\ Huaihe Hospital of Henan University \\ Xia Xu ( $\nabla$ xiaxu_1973@163.com ) \\ Huaihe Hospital of Henan University
}

Research

Keywords:

Posted Date: June 22nd, 2020

DOI: https://doi.org/10.21203/rs.3.rs-36518/v1

License: (1) (1) This work is licensed under a Creative Commons Attribution 4.0 International License.

Read Full License 


\section{Abstract}

Chronic obstructive pulmonary disease (COPD) is a common respiratory disease associated with inflammation and oxidative stress. Sappanone A (SA) is a homoisoflavanone that has been proven to have anti-inflammatory and anti-oxidant effects. However, the role of SA in COPD remains unclear. Thus, the present study was aimed to evaluate the beneficial effect of SA on COPD in vitro. The human bronchial epithelial cells were exposed to $5 \%$ cigarette smoke extracts (CSE) to induce an in vitro model of COPD. Our results showed that SA treatment significantly attenuated the CSE-caused induction of ROS and reduction of SOD and GPx activities in 16HBE cells. In addition, SA inhibited the production of inflammatory cytokines IL-6, IFN- $\gamma$, and TNF- $a$ in CSE-stimulated 16HBE cells. Moreover, the CSEstimulated cell apoptosis of $16 \mathrm{HBE}$ cells were abrogated by SA. Furthermore, we observed that SA treatment greatly promoted the activation of $\mathrm{Nrf2/HO}-1$ signaling pathway, as well as inhibited the activation of TLR4/NF-KB signaling pathway in CSE-stimulated 16HBE cells. Subsequent rescue assay revealed that the protective effects of SA on CSE-stimulated 16HBE cells were reversed by Nrf2 knockdown or TLR4 overexpression. Taken together, these findings demonstrated that SA inhibits oxidative stress, inflammation and apoptosis in CSE-induced human bronchial epithelial cells through regulating Nrf2/HO-1 and TLR4/NF-KB signaling pathways.

\section{Background}

Chronic obstructive pulmonary disease (COPD) is a common respiratory disease that kills more than 3 million people worldwide every year [1]. Despite progress in the treatment of symptoms and prevention of acute exacerbations, limited approaches have been found to ameliorate the progression or affect mortality. COPD presents a significant challenge to the health worldwide and remains the fourth leading cause of death $[2,3]$. Thus, a better understanding of the complex mechanisms resulting in COPD is needed for exploring therapeutic methods.

Cigarette smoking is the main cause of COPD globally, moreover, exposure to smoke and particles from biomass fuel may become more important than cigarette smoke [4]. COPD is an often-progressive inflammatory disease of the airways caused by cigarette smoke. In patients with COPD, the normal inflammatory response in lung tissue is amplified because of harmful factors [5]. The injury of airway epithelial cells releases various endogenous intracellular molecules or danger-associated molecular patterns, which trigger a nonspecific inflammatory response [6,7]. Furthermore, reactive oxygen species (ROS) and oxidative stress have been implicated in the pathogenesis of COPD $[8,9]$. The increased inflammatory response and oxidative stress in airway epithelial cells lead to the cell apoptosis and contribute to lung injury.

Sappanone A (SA) is a homoisoflavanone extracted from the heartwood of Caesalpinia sappan L. Experimental studies have been demonstrated that SA possesses powerful pharmacological activities and potential prospect on the treatment of inflammatory diseases, osteoclastogenesis and melanogenesis $[10,11]$. SA alleviates hypoxia/reoxygenation $(\mathrm{I} / \mathrm{R})$-induced cardiomyocytes injury through 
inhibition of mitochondrial apoptosis and activation of PI3K/Akt/Gsk-3ß pathway [12]. SA protects against myocardial I/R injury via coordinating the cellular antioxidant defenses and maintaining the redox balance [13]. SA inhibits RANKL-induced osteoclastogenesis in bone marrow macrophages (BMMs) and prevents inflammation-mediated bone loss by inhibiting the AKT/GSK-3 $\beta$ signaling pathway [10]. In addition, SA attenuates allergic airway inflammation in ovalbumin-induced asthma by activating the Nrf2 signaling pathway [14]. Considering SA possesses powerful anti-oxidative and anti-inflammatory activities, we assumed that SA might have a potential use for preventing against COPD.

In this study, we examined the effects of SA on bronchial epithelial cells exposed to cigarette smoke extracts (CSE). The results showed that SA exhibited beneficial effects on CSE-induced oxidative stress, inflammation and apoptosis in bronchial epithelial cells, which were mediated by the TLR4/NF-KB and Nrf2/HO-1 signaling pathways.

\section{Materials And Methods}

\subsection{Cell culture}

The human bronchial epithelial cell line 16HBE cells (Cell Bank of Chinese Academy of Sciences, Shanghai, China) were cultured in RPMI-640 medium (Hyclone, Logan, UT, USA). The medium was supplemented with $10 \%$ fetal bovine serum (Gibco, Carlsbad, CA, USA) and 1\% antibiotics (Gibco). The $16 \mathrm{HBE}$ cells were cultivated at $37^{\circ} \mathrm{C}$ in a humidified atmosphere of $5 \% \mathrm{CO}_{2}$.

\subsection{Cell transfection}

To obtained Nrf2-knockdown 16HBE cells, the 16HBE cells were transfected with si-Nrf2 (Invitrogen, Carlsbad, CA, USA). The $16 \mathrm{HBE}$ cells transfected with si-NC were used as negative controls. To generate the TLR4-overexpressing $16 \mathrm{HBE}$ cells, the $16 \mathrm{HBE}$ cells were transfected with pcDNA3.0-TLR4. The 16HBE cells transfected with pcDNA3.0 were used as negative controls. After post transfection, the transfection efficiency was confirmed by western blot analysis.

\subsection{Preparation of CSE}

CSE was prepared from Daqingshan cigarettes (Tar: 12.5, Hohhot Cigarette Factory, Hohhot, China; model group) as previously described [15]. A $1 \mathrm{~cm}$ cigarette without filter was combusted with a variable speed pump (Thermo Fisher Scientific, Waltham, MA, USA). The smoke was bubbled through $15 \mathrm{ml}$ doubledistilled water and the solution was filtered through a $0.22-\mu \mathrm{m}$ pore filter to remove large particles. This resulting suspension was used as $100 \%$ CSE mother solution and then diluted to the concentration of $5 \%$ CSE with culture medium.

\subsection{Cell viability assay}

The cell viability of $16 \mathrm{HBE}$ cells was evaluated by MTT assay, which depends on a colorimetric method. The cells were treated with $10 \mu \mathrm{l}$ of MTT reagent $(5 \mathrm{mg} / \mathrm{ml})$. After incubation for $4 \mathrm{~h}$ at $37^{\circ} \mathrm{C}$, the culture 
medium was replaced with $100 \mu \mathrm{l}$ of DMSO. Finally, sample absorbance was analyzed by the ELISAreader (Bio-Tek Instruments, Winooski, VT, USA) at a wavelength of $490 \mathrm{~nm}$.

\subsection{Measurement of ROS production}

ROS production was assessed using the 2',7'-dichlorodihydrofluorescein diacetate $\left(\mathrm{H}_{2}\right.$ DCF-DA), which can diffuse into the cells and go through deacetylation and oxidization by cellular enzymes into $2{ }^{\prime}, 7^{\prime}$ dichlorofluorescein (DCF) in the presence of ROS. In brief, the treated 16HBE cells were collected and incubated with $\mathrm{H}_{2}$ DCF-DA for 30 min at room temperature in the dark. Then, the cells were washed for three times and the fluorescence intensity in each group was analyzed using flow cytometry with the excitation wavelength $488 \mathrm{~nm}$ and the emission wavelength $525 \mathrm{~nm}$.

\subsection{Detection of glutathione peroxidase (GPX) and superoxide dismutase (SOD) activities}

After treatment, the activities of GPx and SOD in the supernatants were measured using corresponding commercial kits (Beyotime Biotechnology, Shanghai, China) according to the manufacturer's protocol.

\subsection{Quantitative real-time PCR (qRT-PCR)}

Total RNA was isolated from $16 \mathrm{HBE}$ cells by the single-step method using Trizol reagent (Applied Biosystems, Waltham, MA, USA) following the manufacturer's instructions. Then the total RNA was transcribed with CDNA transcription reagent (Applied Biosystems) to obtain CDNA. The expressions of IL6, IFN- - , and TNF- $\alpha$ at mRNA levels were determined by qRT-PCR using SYBR Green Master Mix (Applied Biosystems) with the use of an ABI PRISM 7500 Sequence Detection System (Applied Biosystems). The levels of target mRNA were normalized to $\beta$-actin mRNA, which is an internal control. $\beta$-actin primers were: forward: 5'-ATCA CCAT TGGC AATG AGCG-3' and reverse: 5'-TTGA AGGT AGTT TCGT GGAT-3'; TNFa primers were: forward: 5'-TTC TGT CTA CTG AAC TTC GGG GTG ATC GGT CC-3' and reverse: 5'-GTA TGA GAT AGC AAA TCG GCT GAC GGT GTG GG; IFN-y primers were: forward: 5'-ACT GGC AAA AGG ATG GTG AC-3' and reverse: 5'-TGA GCT CAT TGA ATG CTT GG; IL-6 primers were: forward: 5'-AAC GAT GAT GCA CTT GCA GA-3' and reverse: 5'-GAG CAT TGG AAA TTG GGG TA-3'.

\subsection{Western blot analysis}

After addition with appropriate amount of lysis buffer (Beyotime Biotechnology, Shanghai, China) and incubation for $30 \mathrm{~min}$, the resultant lysis solution was collected and centrifugated at 12,000 rpm for 15 min at $4^{\circ} \mathrm{C}$. The supernatant was collected for the determination of protein concentration by the BCA method. The protein samples were subjected to denaturation in the $98^{\circ} \mathrm{C}$ metal bath for 5 min and then separated by $12 \%$ SDS-PAGE. Afterwards, the proteins were transferred onto the PVDF membrane, followed by blocking with $5 \%$ skim milk for $1 \mathrm{~h}$ and incubation with the corresponding primary antibodies overnight at $4^{\circ} \mathrm{C}$. The primary antibodies against bax, bcl-2, cleaved caspase-3, nuclear Nrf2, HO-1, TLR4, p65, p-p65 and IKBa were used in this study (Abcam, Cambridge, MA, USA). Next, the membrane was incubated with secondary antibody at room temperature for $1 \mathrm{~h}$. The secondary antibody was used at a 
dilution of 1: 3000 (Abcam). The bands on the membrane were then visualized by the chemiluminescence method using chemiluminescence detection system (Amersham Biosciences, Piscataway, NJ, USA). The gray value was analyzed via the Image-J software (National Institutes of Health, NIH, Bethesda, MD, USA).

\subsection{Cell apoptosis assay}

Caspase 3 activity in cellular lysates was assessed using the Caspase 3 Activity Assay Kit purchased from the Beyotime Institute of Biotechnology. Briefly, each sample was incubated with caspase 3 substrate (Ac-DEVD-pNA) at $37^{\circ} \mathrm{C}$ for a further $2 \mathrm{~h}$. Finally, the reaction products were measured with Microplate Spectrophotometer (Thermo Fisher Scientific) at an absorbance of $405 \mathrm{~nm}$.

\subsection{ELISA}

Supernatants from experimental cultures of $16 \mathrm{HBE}$ cells were collected and stored at $-80^{\circ} \mathrm{C}$ until use. The levels of IL-6, IFN- $\gamma$, and TNF-a in the supernatants were determined by using ELISA kits (R\&D Systems, Minneapolis, MN, USA) in accordance with the manufacturer's instructions. Finally, the detection of absorbance at $450 \mathrm{~nm}$ was conducted by using a microplate reader (Bio-Tek).

\subsection{Statistical analysis}

Data are expressed as means \pm SEM. Experiments with multiple comparisons were analyzed by one-way ANOVA followed by Tukey post hoc test using IBM SPSS statistics version 22.0. A $p$ value less than 0.05 $(p<0.05)$ was regarded as statistically significant.

\section{Results}

\subsection{Effect of SA on cell viability in 16HBE cells}

Firstly, the effect of the different doses of $S A(0,5,10,20$ and $40 \mu \mathrm{M})$ pretreatment on the cell viability of $16 \mathrm{HBE}$ cells was examined. As shown in Figure 1, cell viability of $16 \mathrm{HBE}$ cells was not significantly changed after treatment with 5,10 or $20 \mu \mathrm{M} \mathrm{SA}$. Thus, the concentrations of 5,10 and $20 \mu \mathrm{M}$ were selected for the following experiments.

\subsection{SA inhibited oxidative stress in CSE-stimulated 16HBE cells}

Next, to elucidate the effect of SA on CSE-stimulated oxidative stress in 16HBE cells, the production of ROS was measured. We found that SA pretreatment inhibited CSE-induced ROS production in $16 \mathrm{HBE}$ cells 
in a dose-dependent manner (Figure 2A). Besides, the activities of GPx and SOD were also detected to reflect oxidative stress. As indicated in Figures 2B and 2C, CSE-caused decreases in GPx and SOD activities were dose-dependently prevented by SA pretreatment.

\subsection{SA inhibited the levels of pro-inflammatory cytokines in CSE-stimulated 16HBE cells}

Then we measured the production levels of pro-inflammatory cytokines including IL-6, IFN- - , and TNF- $a$ by ELISA and qRT-PCR, respectively. ELISA results showed that SA significantly inhibited the release of IL6, IFN- $y$, and TNF-a in 16HBE cells exposed to CSE (Figures 3A-3C). Similarly, we also found that the mRNA levels of IL-6, IFN- - , and TNF-a in 16HBE cells were induced by CSE, which were repressed by SA (Figures 3D-3F).

\subsection{SA suppressed cell apoptosis in CSE-stimulated 16HBE cells}

In order to test whether SA affected the CSE-induced cell apoptosis in 16HBE cells, western blot analysis was performed to evaluate the expression levels of bax, bcl-2 and cleaved caspase- 3 . The results of western blot assay indicated that CSE stimulation caused significant increases in bax and cleaved caspase-3 expressions and a decrease in bcl-2 expression. SA treatment was shown to attenuate CSEinduced changes in the expression levels of bax, bcl-2 and cleaved caspase-3 (Figure 4A). Also, caspse-3 activity is an index of oxidative stress increased in CSE-stimulated 16HBE cells. However, SA dosedependently reduced the caspse-3 activity in 16HBE cells exposed to CSE stimulation (Figure 4B).

\subsection{SA decreased TLR4/NF-KB activation in CSE-stimulated 16HBE cells}

The activation of TLR4/NF-KB signaling pathway has been found to be involved in the lung inflammation [16]. Thus, we investigated whether SA could affect the activation of TLR4/NF-KB signaling pathway. Results from western blot demonstrated that CSE significantly increased the levels of TLR4 and p-p65, as well as decreased the level of IKBa in in 16HBE cells; however, SA suppressed the CSE-induced the activation of TLR4/NF-KB signaling pathway in 16HBE cells (Figure 5).

\subsection{TLR4 reversed the anti-inflammatory effect of SA in CSE-stimulated 16HBE cells}

Finally, we confirmed the role of TLR4/NF-KB signaling pathway in the anti-inflammatory effect of SA through transfection with TLR4-overexpressing plasmid pcDNA3.0-TLR4. Results from western blot showed that the protein expression level of TLR4 was upregulated by pcDNA3.0-TLR4 in 16HBE cells cotreated with SA and CSE (Figure 6A and 6B). As indicated in Figures $6 \mathrm{C}-6 \mathrm{E}$, the inhibitory effects of SA on the secretion of IL-6, IFN- $\gamma$, and TNF-a were reversed by overexpression of TLR4 in 16HBE cells exposed to CSE.

\subsection{SA induced the activation of Nrf2/HO-1 pathway in CSE-stimulated 16HBE cells}

Nrf2/HO-1 signaling pathway plays a pivotal role in cellular defense against oxidative stress through induction of antioxidant genes [17]. So, we investigated the effect of SA on Nrf2/HO-1 signaling pathway 
in $16 \mathrm{HBE}$ cells exposed to CSE. The results showed that CSE treatment significantly inhibited the protein levels of Nrf2 and HO-1. However, the SA-treated groups exhibited enhanced increase in the Nrf2 and HO1 expression levels (Figure 7).

\subsection{Knockdown of Nrf2 abrogated the protective effect of SA on CSE-stimulated 16HBE cells}

Because the activation of Nrf2/HO-1 signaling pathway was found in SA-treated $16 \mathrm{HBE}$ cells, we next examined whether Nrf2 blockade could affect the protective effect of SA through transfection with siNrf2. As shown in Figure 8A and 8B, we observed that knockdown of Nrf2 significantly reduced the protein level of Nrf2 in 16HBE cells co-treated with SA and CSE. In addition, we observed that the protective effects of SA on ROS production and caspase-3 activity were reversed by Nrf2 knockdown in 16HBE cells exposed to CSE (Figures 8C-8D).

\section{Discussion}

Our findings clearly demonstrated that CSE in all concentrations generated oxidative stress, inflammation and cell apoptosis in 16HBE cells. The results of this study also proved that SA attenuated oxidative stress, inflammation and apoptosis in CS -induced 16HBE cells via regulating Nrf2/HO-1 and TLR4/NF-KB signaling pathways.

Cigarette smoke is made up of thousands of toxic chemicals that could cause several pathological events including oxidative stress, inflammation and cell toxicity [18]. All of these changes may lead to the dysregulation of pulmonary cells and exacerbate the development of lung injury. Numerous studies have identified that long-term exposure to cigarette smoke is one of the main causes of COPD [19]. Chronic bronchitis and emphysema are the most common disorders responsible for COPD [20]. In COPD, chronic inflammation mainly entails the infiltration of inflammatory cells, such as neutrophils, macrophages, and $\mathrm{CD}^{+} \mathrm{T}$ lymphocytes into the small airways, leading to the chronic bronchitis [21]. In fact, pathological alterations in bronchial epithelial cells may cause a loss of small airway destruction, which contribute to lung injury in COPD [22]. Herein, the human bronchial epithelial cell line 16HBE was exposed to $10 \%$ CSE to induce an in vitro model of COPD, and thus investigated the beneficial effects of SA on the CSEinduced cell injury in $16 \mathrm{HBE}$ cells.

It is well documented that anti-inflammatory supplements have a direct action on attenuating the progression of COPD [23]. Considering the anti-inflammatory effect of SA, we explored the role of SA in CSE-induced inflammatory response in 16HBE cells. The results of the present study demonstrated that exposure to CSE caused significant increase in the release of pro-inflammatory cytokines including IL-6, IFN- $y$, and TNF-a. However, treatment with SA attenuated CSE-induced inflammatory response in a dosedependent manner. The results indicated that SA might exert anti-inflammatory activity in CSE-induced $16 \mathrm{HBE}$ cells. It has been demonstrated that the mechanism of COPD is complex and involves a number of pathways. Most importantly, the inflammatory signaling exhibit the vital effects on pulmonary 
inflammation, which may provide major therapeutic targets in COPD $[24,25]$. TLR4 is a pattern recognition receptor that is typically known to play a major role in generating an inflammatory response by way of activating various essential downstream components [26]. NF-KB has been observed as an important downstream transcriptional factor of TLR4, which is crucial in the development of inflammatory diseases through regulation of the gene expression of inflammation mediums such as IL-1 $\beta$ and TNF-a [27]. Several studies highlight the role of TLR4/NF-KB signaling in the inflammatory lung diseases, such as asthma and COPD $[28,29]$. We found that exposure to CSE significantly induced the activation of TLR4/NF-KB signaling pathway in 16HBE cells. While treatment with SA inhibited the CSE-induced activation of TLR4/NF-KB signaling pathway. Moreover, restoration of TLR4 reversed the protective effects of SA on CSE-induced cell injury in 16HBE cells. These findings suggested that the antiinflammatory effect of SA might be mediated by the inhibition of TLR4/NF-KB signaling pathway.

In addition to inflammation, several promising mechanisms including oxidative stress and protease/antiprotease imbalance have also been suggested for the small airway destruction and structural changes $[30,31]$. Oxidative stress is a purported contributor for COPD through activating pro-inflammatory cytokine transcription [32]. The accelerated oxidative stress and inflammation may finally result in cell apoptosis of small airway epithelial cells. The results of our study also proved that treatment with SA prevented CSE-induced oxidative stress with decreased level of ROS and increased activities of GPx and SOD. Besides, the CSE-induced apoptosis was also mitigated by SA, as proved by the increased expression level of bcl-2, and deceased expression level of bax and decreased caspase-3 activity. The nuclear factor, Nrf2, is a basic leucine zipper (bZIP) protein activated by pro-oxidant, oxidant and antioxidant agents. Under pathological condition, these agents allow Nrf2 to dissociate from Kelch-like ECH associated protein 1 (Keap1), which is a cytoplasmic inhibitor of Nrf2 [33]. Then the separated Nrf2 translocates to the nucleus and regulates the activation of cellular rescue pathways against oxidative injury, inflammation, and apoptosis [34]. Moreover, we also found that SA enhanced the activation of Nrf2/HO-1 signaling pathway in 16HBE cells exposed to CSE. Knockdown of Nrf2 resulted in an inhibitory effect on the beneficial effects of SA on 16HBE cells exposed to CSE. Collectively, Nrf2/HO-1 signaling pathway played a critical role in the anti-oxidative and anti-apoptotic activities of SA in CSE-induced 16HBE cells.

\section{Conclusion}

In conclusion, our results proved that SA exerted anti-inflammatory, anti-oxidative and anti-apoptotic activities in CSE-induced 16HBE cells via regulating TLR4/NF-KB and Nrf2/HO-1 signaling pathways. In light of this findings, we hypothesized that SA might serve as a potent therapeutic agent for the treatment of COPD.

\section{Declarations}




\section{Conflict of interest statement}

No conflict of interests.

Ethics approval and consent to participate

Not applicable.

Consent for publication

Not applicable.

\section{Availability of data and material}

Not applicable.

\section{Authors' contributions'}

The research was conceived and designed by Xia Xu. The experiments was carried out by Shanshan Wang and Hongli Li.The manuscript was wrote by Yan Zhang.

\section{Funding}

None

Acknowledgements

None

\section{References}

1. Rabe KF, Watz H. Chronic obstructive pulmonary disease. Lancet 2017;389: 1931-1940.

2. Viegi G, Scognamiglio A, Baldacci S, Pistelli F, Carrozzi L. Epidemiology of chronic obstructive pulmonary disease (COPD). Respiration 2001;68: 4-19.

3. Pauwels RA, Rabe KF. Burden and clinical features of chronic obstructive pulmonary disease (COPD). Lancet 2004;364: 613-620.

4. Duffy SP, Criner GJ. Chronic Obstructive Pulmonary Disease: Evaluation and Management. Med Clin North Am 2019;103: 453-461.

5. Eapen MS, Myers S, Walters EH, Sohal SS. Airway inflammation in chronic obstructive pulmonary disease (COPD): a true paradox. Expert Rev Respir Med 2017;11: 827-839. 
6. Ling Y, Wang X, Jin M. Role of Airway Epithelial Cells in Development of Chronic Obstructive Pulmonary Disease. J Epithelial Biol Pharmacol 2009;2: 44-50.

7. Randell SH. Airway epithelial stem cells and the pathophysiology of chronic obstructive pulmonary disease. Proc Am Thorac Soc 2006;3: 718-725.

8. Riou M, Alfatni A, Charles AL, Andres E, Pistea C, Charloux A, et al. New Insights into the Implication of Mitochondrial Dysfunction in Tissue, Peripheral Blood Mononuclear Cells, and Platelets during Lung Diseases. J Clin Med 2020;9: E1253.doi: 10.3390/jcm9051253.

9. Boukhenouna S, Wilson MA, Bahmed K, Kosmider B. Reactive Oxygen Species in Chronic Obstructive Pulmonary Disease. Oxid Med Cell Longev 2018;2018: 5730395.

10. Choo YY, Tran PT, Min BS, Kim O, Nguyen HD, Kwon SH, et al. Sappanone A inhibits RANKL-induced osteoclastogenesis in BMMs and prevents inflammation-mediated bone loss. Int Immunopharmacol 2017; 52: 230-237.

11. Chang TS, Chao SY, Ding HY. Melanogenesis inhibition by homoisoflavavone sappanone A from Caesalpinia sappan. Int J Mol Sci 2012;13: 10359-10367.

12. Shi X, Tao G, Ji L, Tian G. Sappanone A alleviates hypoxia/reoxygenation-induced cardiomyocytes injury through inhibition of mitochondrial apoptosis and activation of PI3K-Akt-Gsk-3beta pathway. Biosci Rep 2020;40

13. Shi X, Tao G, Ji L, Tian G. Sappanone A Protects Against Myocardial Ischemia Reperfusion Injury by Modulation of Nrf2. Drug Des Devel Ther 2020;14: 61-71.

14. Liu X, Yu D, Wang T. Sappanone A Attenuates Allergic Airway Inflammation in Ovalbumin-Induced Asthma. Int Arch Allergy Immunol 2016;170: 180-186.

15. Farid M, Kanaji N, Nakanishi M, Gunji Y, Michalski J, Iwasawa S, et al. Smad3 mediates cigarette smoke extract (CSE) induction of VEGF release by human fetal lung fibroblasts. Toxicol Lett 2013;220: 126-134.

16. Zhang ZM, Wang YC, Chen L, Li Z. Protective effects of the suppressed NF-kappaB/TLR4 signaling pathway on oxidative stress of lung tissue in rat with acute lung injury. Kaohsiung J Med Sci 2019;35: 265-276.

17. Loboda A, Damulewicz M, Pyza E, Jozkowicz A, Dulak J. Role of Nrf2/HO-1 system in development, oxidative stress response and diseases: an evolutionarily conserved mechanism. Cell Mol Life Sci 2016;73: 3221-3247.

18. Budani MC, Tiboni GM. Ovotoxicity of cigarette smoke: A systematic review of the literature. Reprod Toxicol 2017;72: 164-181.

19. Tamimi A, Serdarevic D, Hanania NA. The effects of cigarette smoke on airway inflammation in asthma and COPD: therapeutic implications. Respir Med 2012;106: 319-328.

20. Forey BA, Thornton AJ, Lee PN. Systematic review with meta-analysis of the epidemiological evidence relating smoking to COPD, chronic bronchitis and emphysema. BMC Pulm Med 2011;11: 36. 
21. Sun G, Stacey MA, Vittori E, Marini M, Bellini A, Kleimberg J, et al. Cellular and molecular characteristics of inflammation in chronic bronchitis. Eur J Clin Invest 1998;28: 364-372.

22. Gao W, Li L, Wang Y, Zhang S, Adcock IM, Barnes PJ, et al. Bronchial epithelial cells: The key effector cells in the pathogenesis of chronic obstructive pulmonary disease? Respirology 2015;20: 722-729.

23. Loukides S, Bartziokas K, Vestbo J, Singh D. Novel anti-inflammatory agents in COPD: targeting lung and systemic inflammation. Curr Drug Targets 2013;14: 235-245.

24. Schuliga M. NF-kappaB Signaling in Chronic Inflammatory Airway Disease. Biomolecules 2015;5: 1266-1283.

25. Aghasafari P, George U, Pidaparti R. A review of inflammatory mechanism in airway diseases. Inflamm Res 2019;68: 59-74.

26. Rogero MM, Calder PC. Obesity, Inflammation, Toll-Like Receptor 4 and Fatty Acids. Nutrients 2018;10:432.

27. Roy A, Srivastava M, Saqib U, Liu D, Faisal SM, Sugathan S, et al. Potential therapeutic targets for inflammation in toll-like receptor 4 (TLR4)-mediated signaling pathways. Int Immunopharmacol 2016;40: 79-89.

28. Chen L, Ran D, Xie W, Xu Q, Zhou X. Cold-inducible RNA-binding protein mediates cold air inducible airway mucin production through TLR4/NF-kappaB signaling pathway. Int Immunopharmacol 2016;39: 48-56.

29. Wu Z, Tan B, Zhang H, Guo Y, Tu Y, Qiu F, et al. Effects of Sodium Houttuyfonate on Pulmonary Inflammation in COPD Model Rats. Inflammation 2017;40: 2109-2117.

30. Fischer BM, Pavlisko E, Voynow JA. Pathogenic triad in COPD: oxidative stress, proteaseantiprotease imbalance, and inflammation. Int J Chron Obstruct Pulmon Dis 2011;6: 413-421.

31. Barnes PJ. Oxidative stress-based therapeutics in COPD. Redox Biol 2020: 101544.

32. Kirkham PA, Barnes PJ. Oxidative stress in COPD. Chest 2013;144: 266-273.

33. Bellezza I, Giambanco I, Minelli A, Donato R. Nrf2-Keap1 signaling in oxidative and reductive stress. Biochim Biophys Acta Mol Cell Res 2018;1865: 721-733.

34. Tonelli C, Chio IIC, Tuveson DA. Transcriptional Regulation by Nrf2. Antioxid Redox Signal 2018;29: 1727-1745.

\section{Figures}




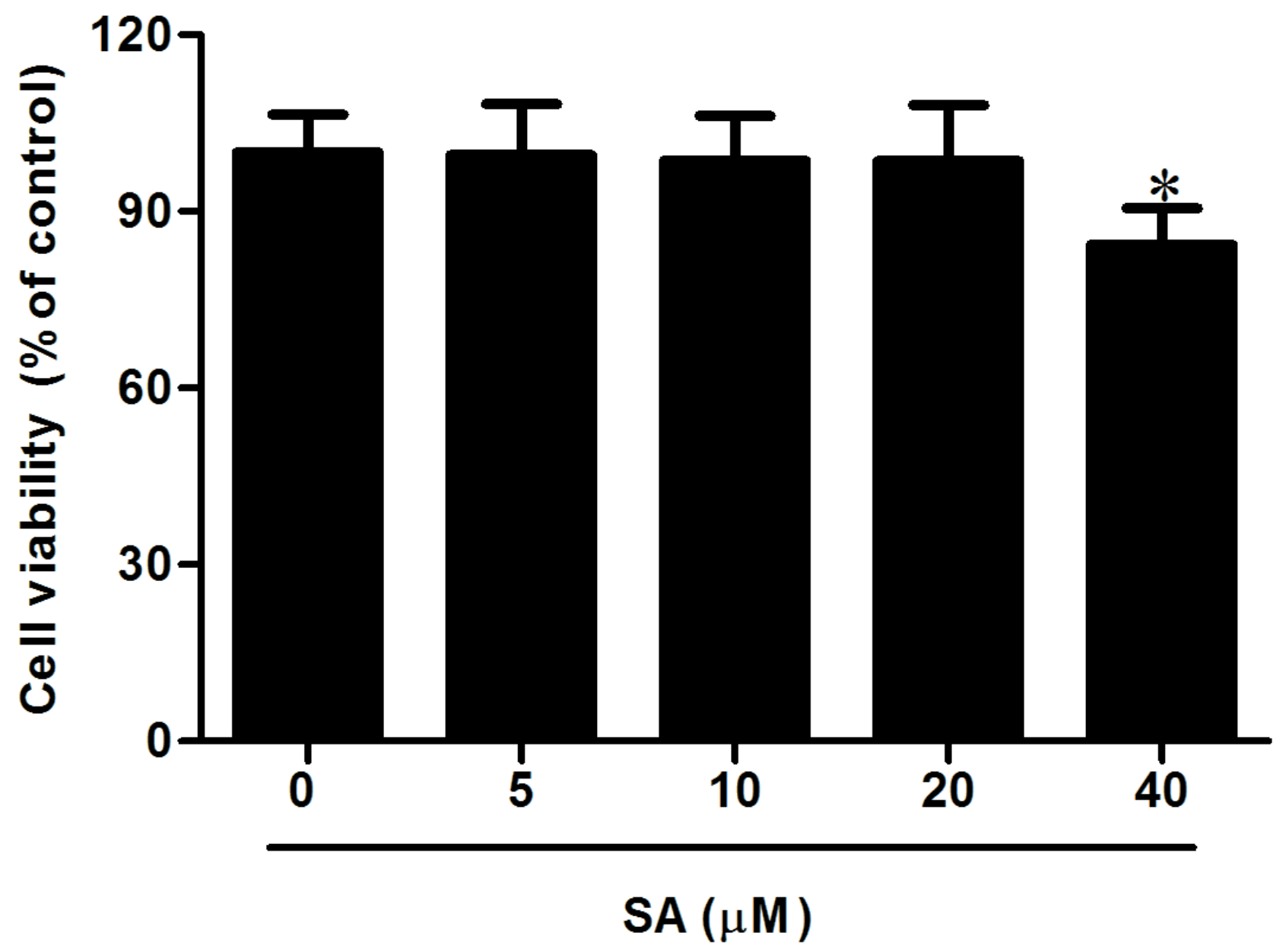

Figure 1

Effects of SA on cell viability in $16 \mathrm{HBE}$ cells. $16 \mathrm{HBE}$ cells were treated with different doses of $\mathrm{SA}(0,5,10$, 20 and $40 \mu \mathrm{M}$ ) for $24 \mathrm{~h}$. Cell viability was examined by MTT assay. ${ }^{*} p<0.05$ compared to normal control $16 \mathrm{HBE}$ cells. 
A

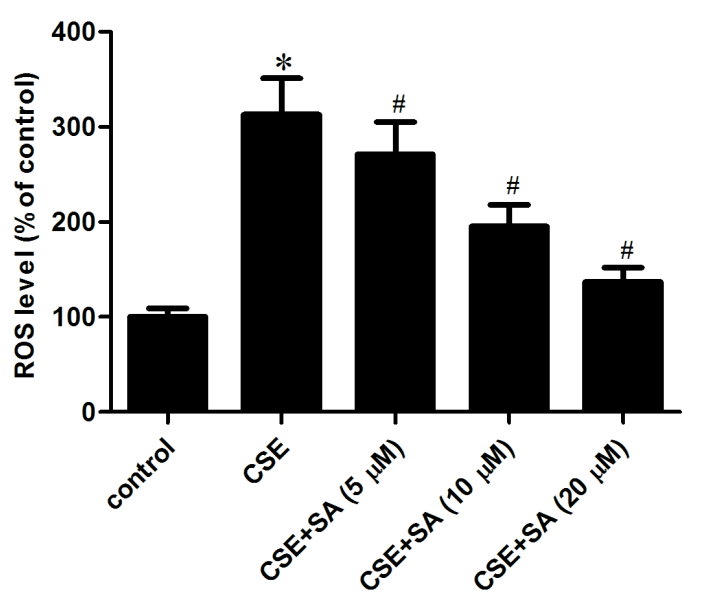

B

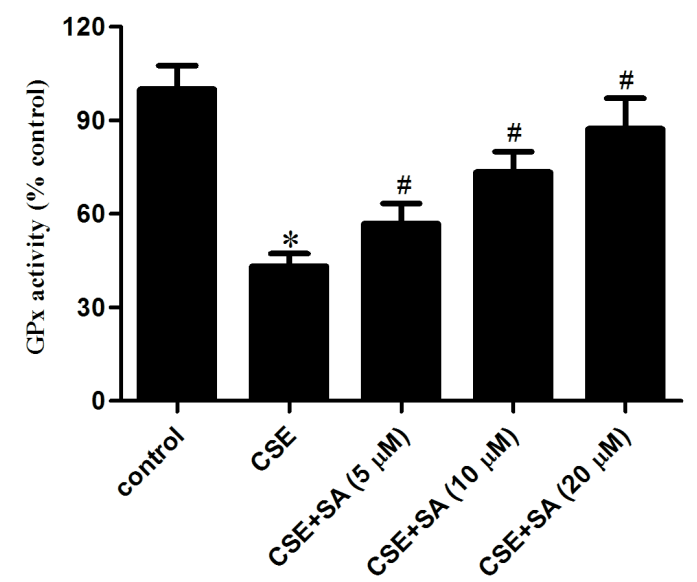

C

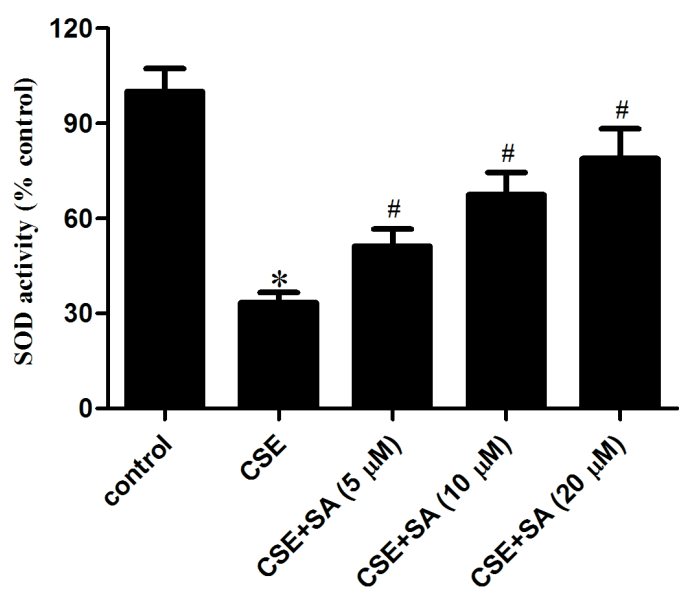

Figure 2

SA inhibited oxidative stress in CSE-stimulated 16HBE cells. 16HBE cells were pretreated with $(5,10$ and $20 \mu \mathrm{M}$ ) for $2 \mathrm{~h}$ and then incubated with 5\% CSE. The results of (A) ROS production, (B) GPx activity and (C) SOD activity in $16 \mathrm{HBE}$ cells. ${ }^{\star} \mathrm{p}<0.05$ compared to control group; $\# \mathrm{p}<0.05$ compared to CSE group. 
A

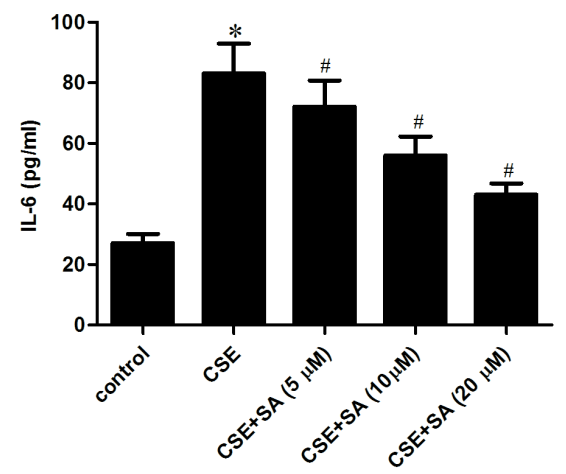

D

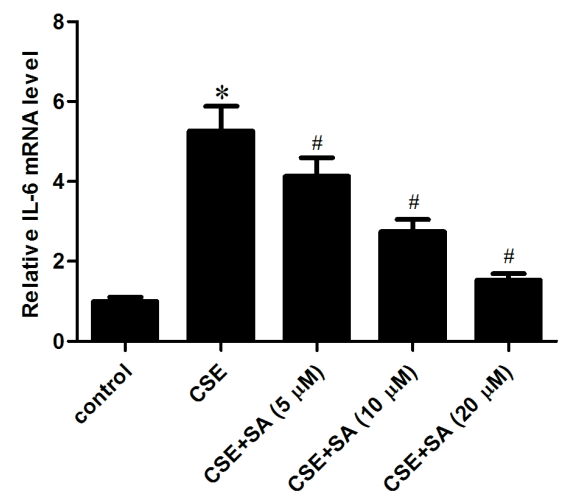

B

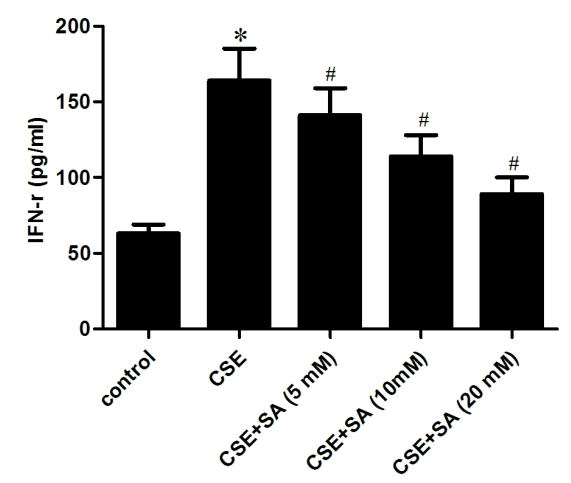

E

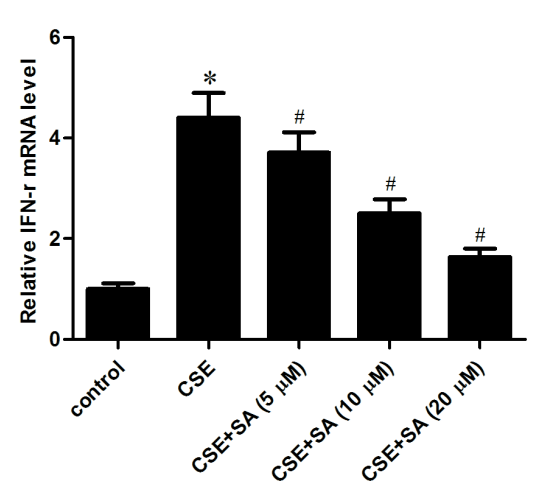

C

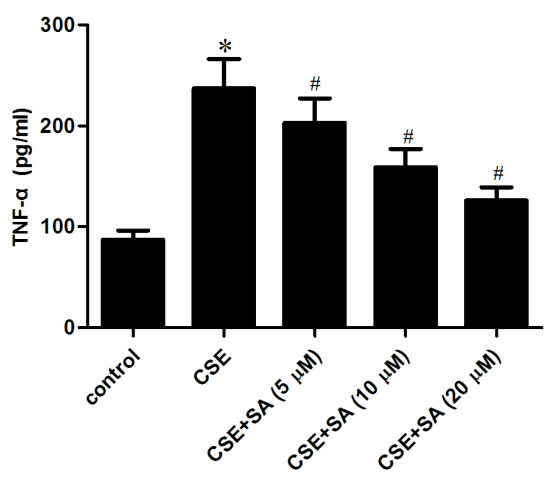

$\mathbf{F}$

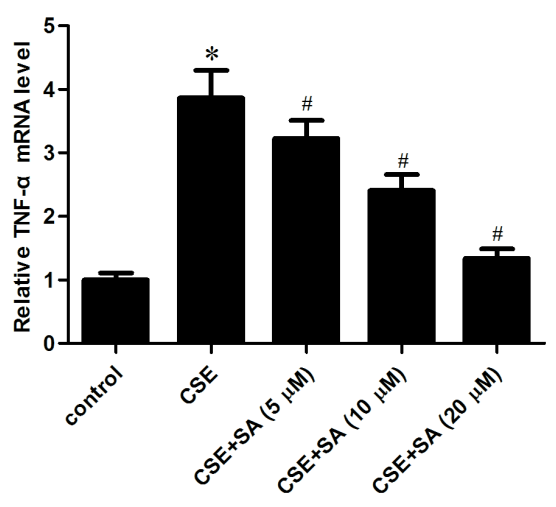

Figure 3

SA inhibited the levels of pro-inflammatory cytokines in CSE-stimulated 16HBE cells. The production levels of pro-inflammatory cytokines including IL-6, IFN- - , and TNF-a were detected by ELISA (A-C) and qRT-PCR (D-F), respectively. * $<<0.05$ compared to control group; $\# p<0.05$ compared to CSE group. 
A
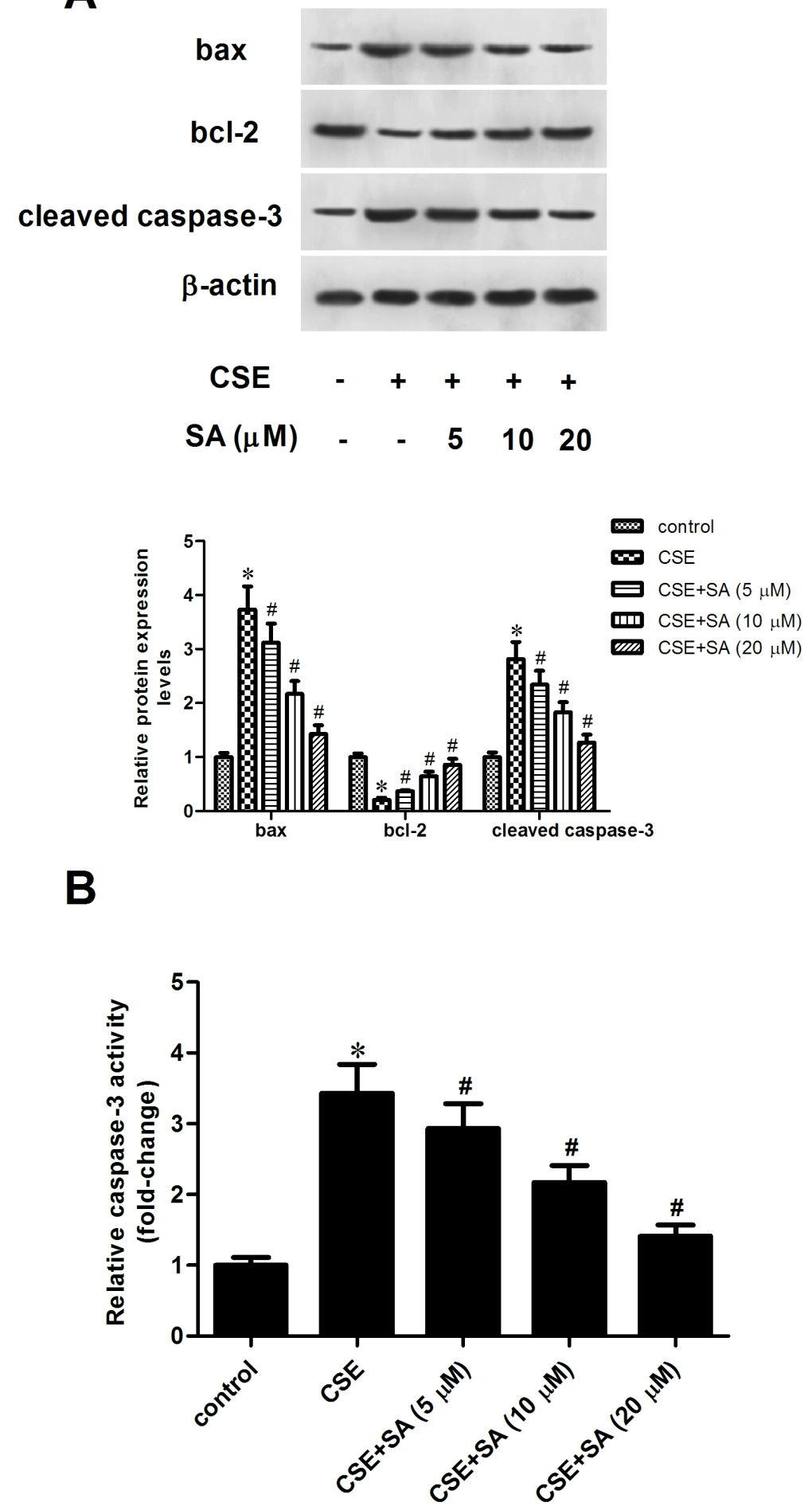

Figure 4

SA suppressed cell apoptosis in CSE-stimulated 16HBE cells. (A) Western blot analysis was performed to evaluate the expression levels of bax, bcl-2 and cleaved caspase-3. Quantitative analysis of bax, bcl-2 and cleaved caspase 3 . (B) Results of caspse-3 activity in $16 \mathrm{HBE}$ cells. ${ }^{*} p<0.05$ compared to control group; $\# p<0.05$ compared to CSE group. 

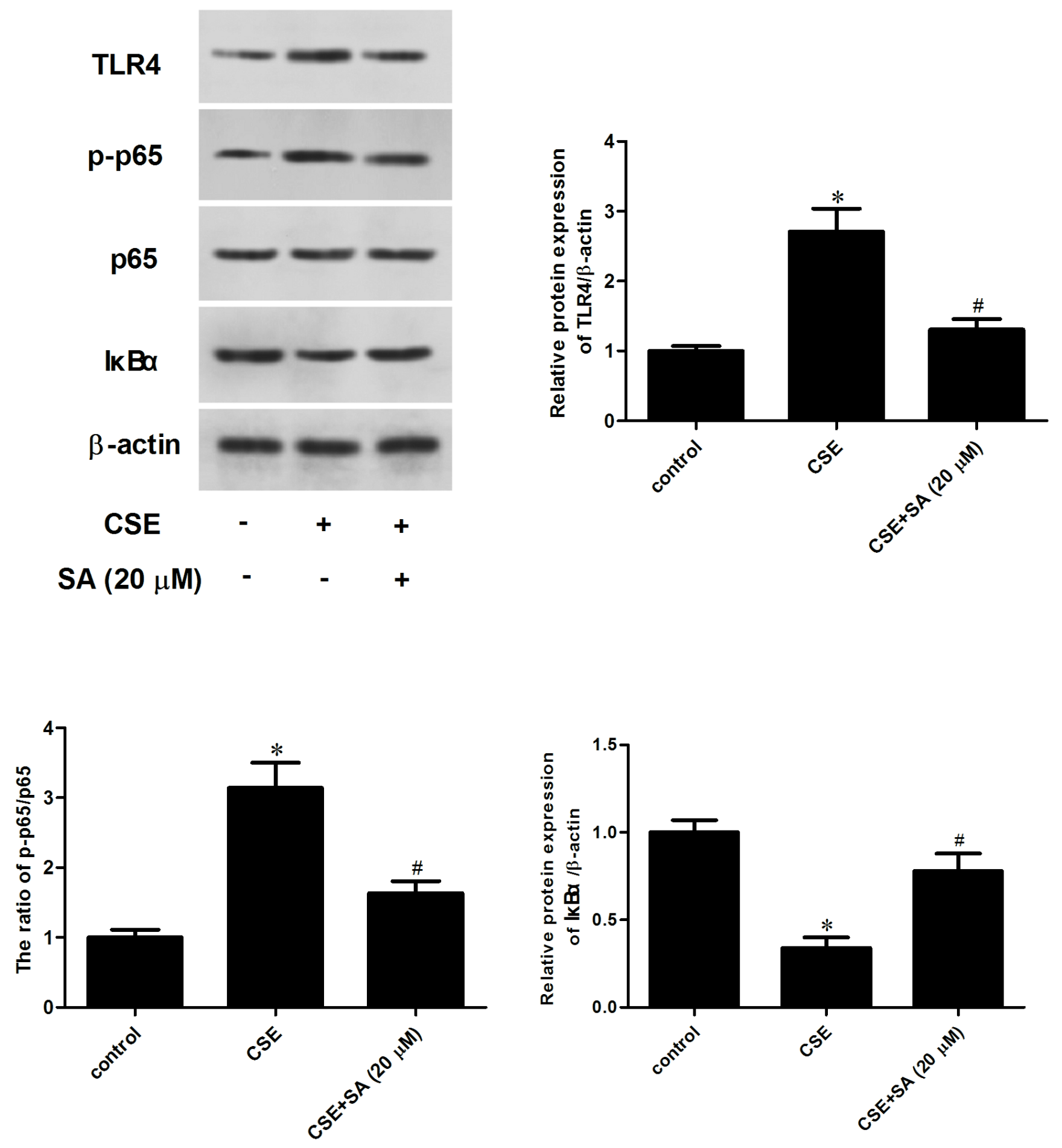

Figure 5

SA decreased TLR4/NF-KB activation in CSE-stimulated 16HBE cells. 16HBE cells were pretreated with 20 $\mu \mathrm{M}$ SA for $2 \mathrm{~h}$, followed by CSE stimulation. Western blot analysis was performed to evaluate the expression levels of TLR4, p65, p-p65 and IKBa. Quantitative analysis of TLR4, p-p65/p65 and IKBa. ${ }^{*} p<0.05$ compared to control group; $\# p<0.05$ compared to CSE group. 
A

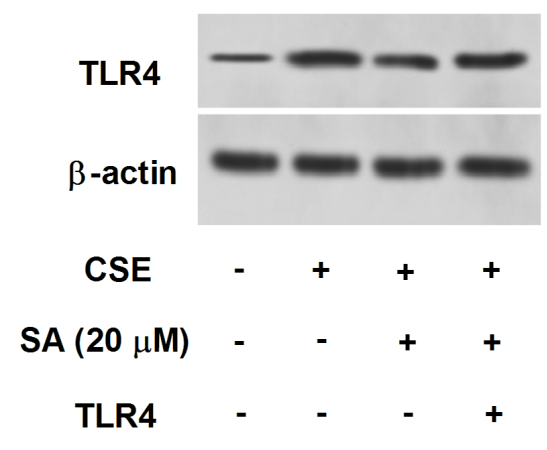

B

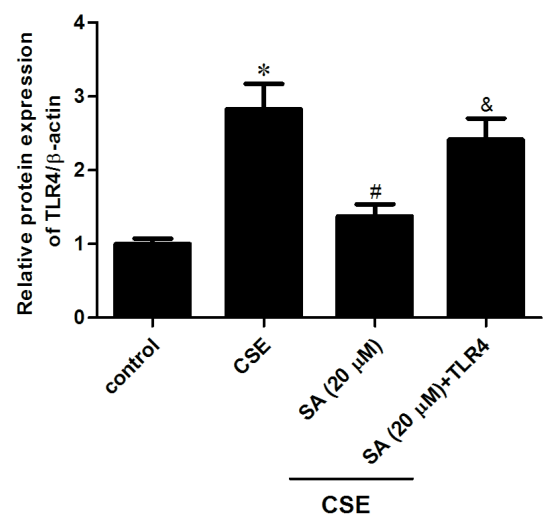

C

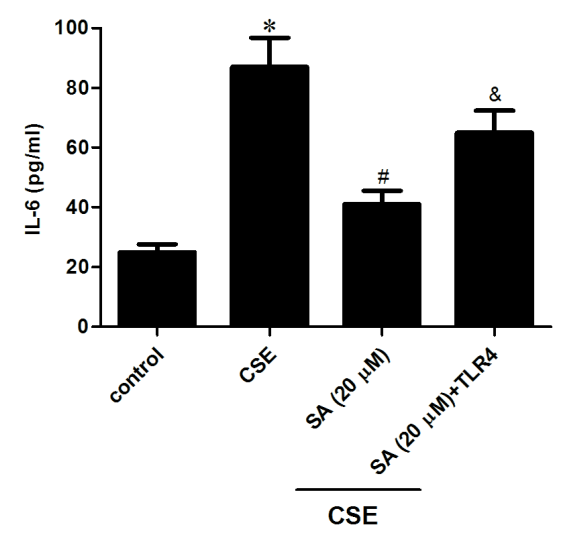

D

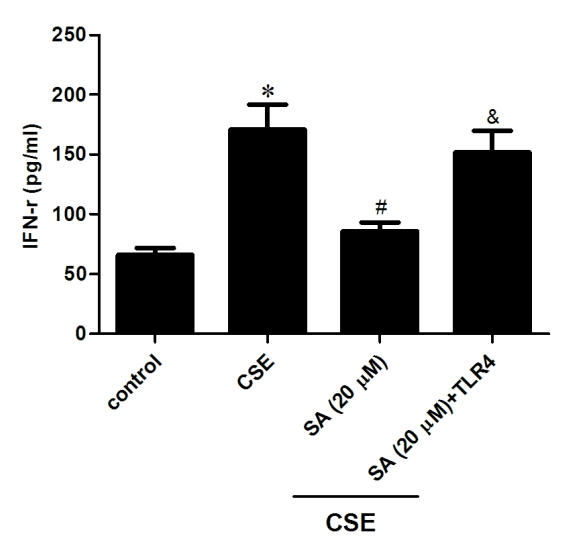

$\mathbf{E}$

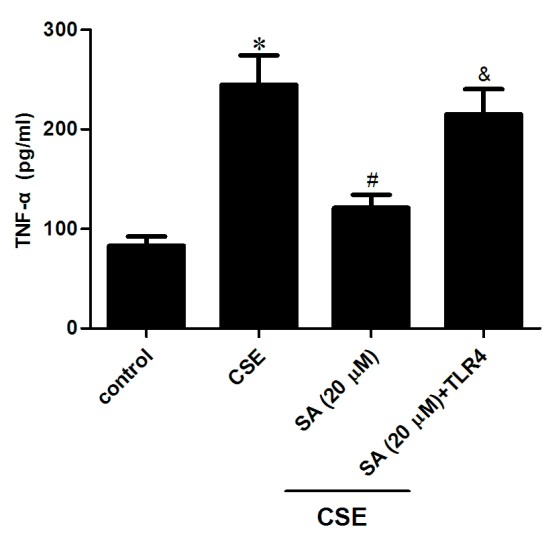

Figure 6

TLR4 reversed the anti-inflammatory effect of SA in CSE-stimulated 16HBE cells. 16HBE cells were transfected with pcDNA3.0-TLR4 for $24 \mathrm{~h}$ in the presence of $20 \mu \mathrm{M}$ SA before CSE stimulation. (A) The protein level of TLR4 was detected by western blot analysis. (B) Quantitative analysis of the relative protein level of TLR4. (C-E) The secretion of IL-6, IFN- $\gamma$, and TNF-a in 16HBE cells. ${ }^{*} p<0.05$ compared to control group; \#p<0.05 compared to CSE group; \&p<0.05 compared to CSE+SA group. 
nuclear Nrf2

lamin B1

HO-1

$\beta$-actin

CSE - + +

$\mathrm{SA}(20 \mu \mathrm{M}) \quad-\quad-\quad+$
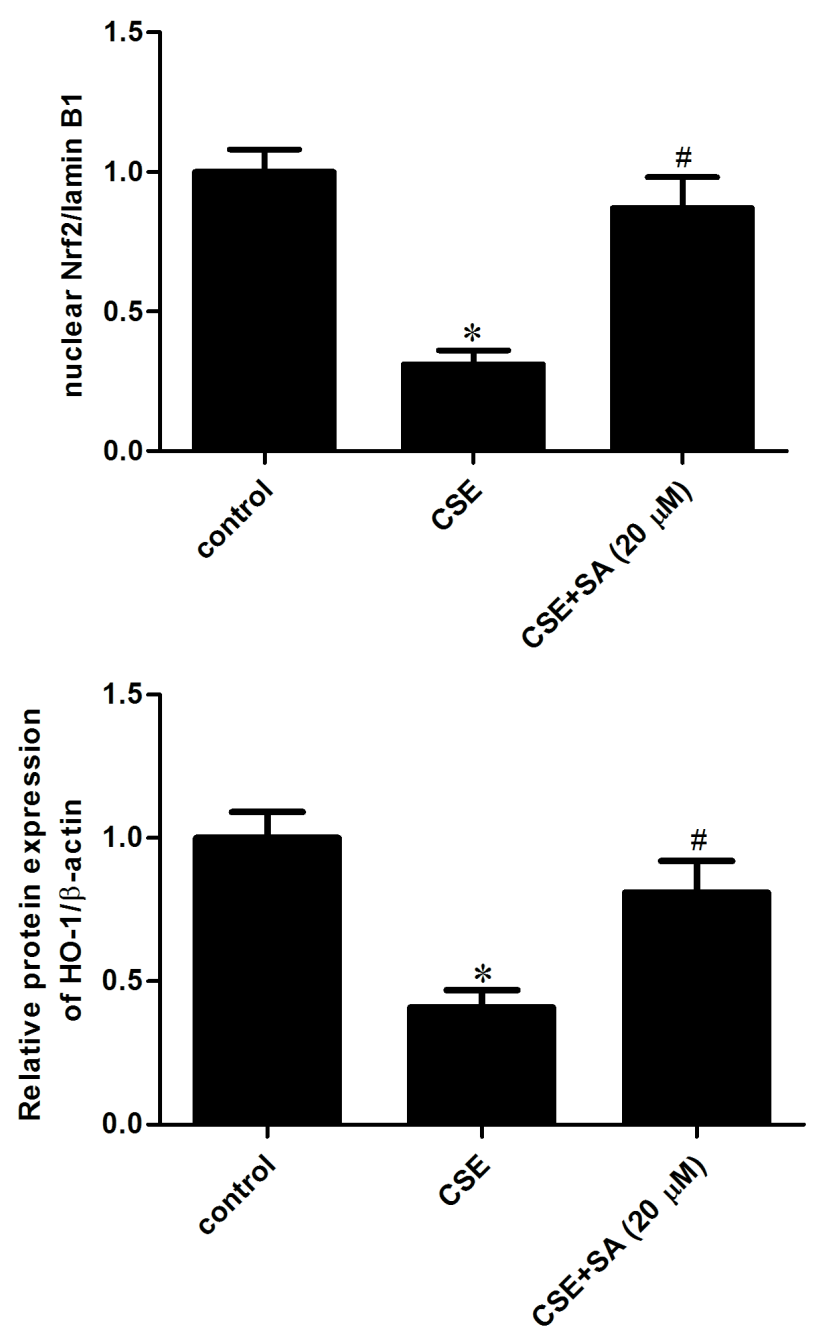

Figure 7

SA induced the activation of Nrf2/HO-1 pathway in CSE-stimulated $16 \mathrm{HBE}$ cells. 16HBE cells were pretreated with $20 \mu \mathrm{M}$ SA for $2 \mathrm{~h}$, followed by CSE stimulation. Western blot analysis was performed to evaluate the expression levels of nuclear Nrf2 and HO-1. Quantitative analysis of nuclear Nrf2 and HO-1. ${ }^{*} p<0.05$ compared to control group; \#p<0.05 compared to CSE group. 
A
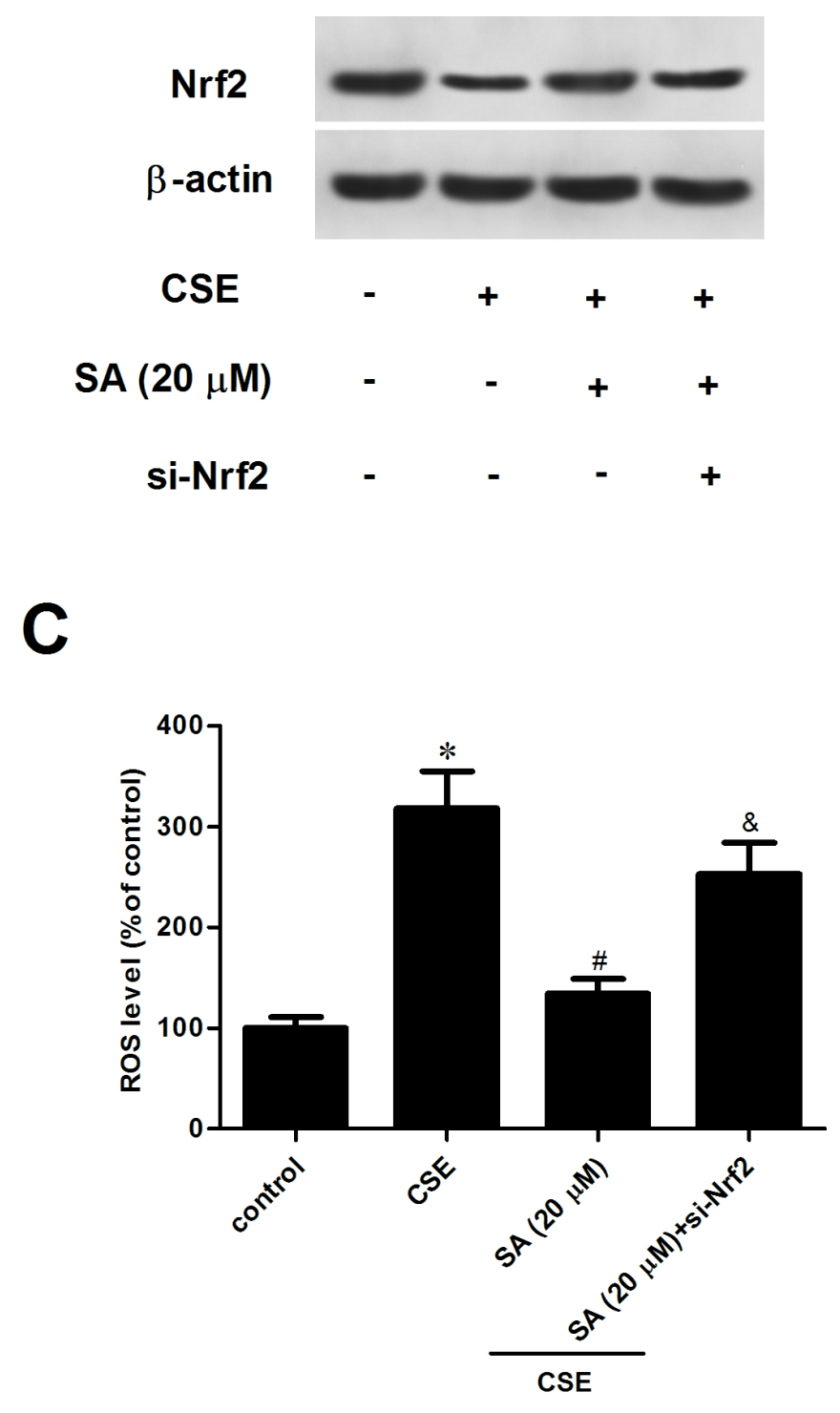

B
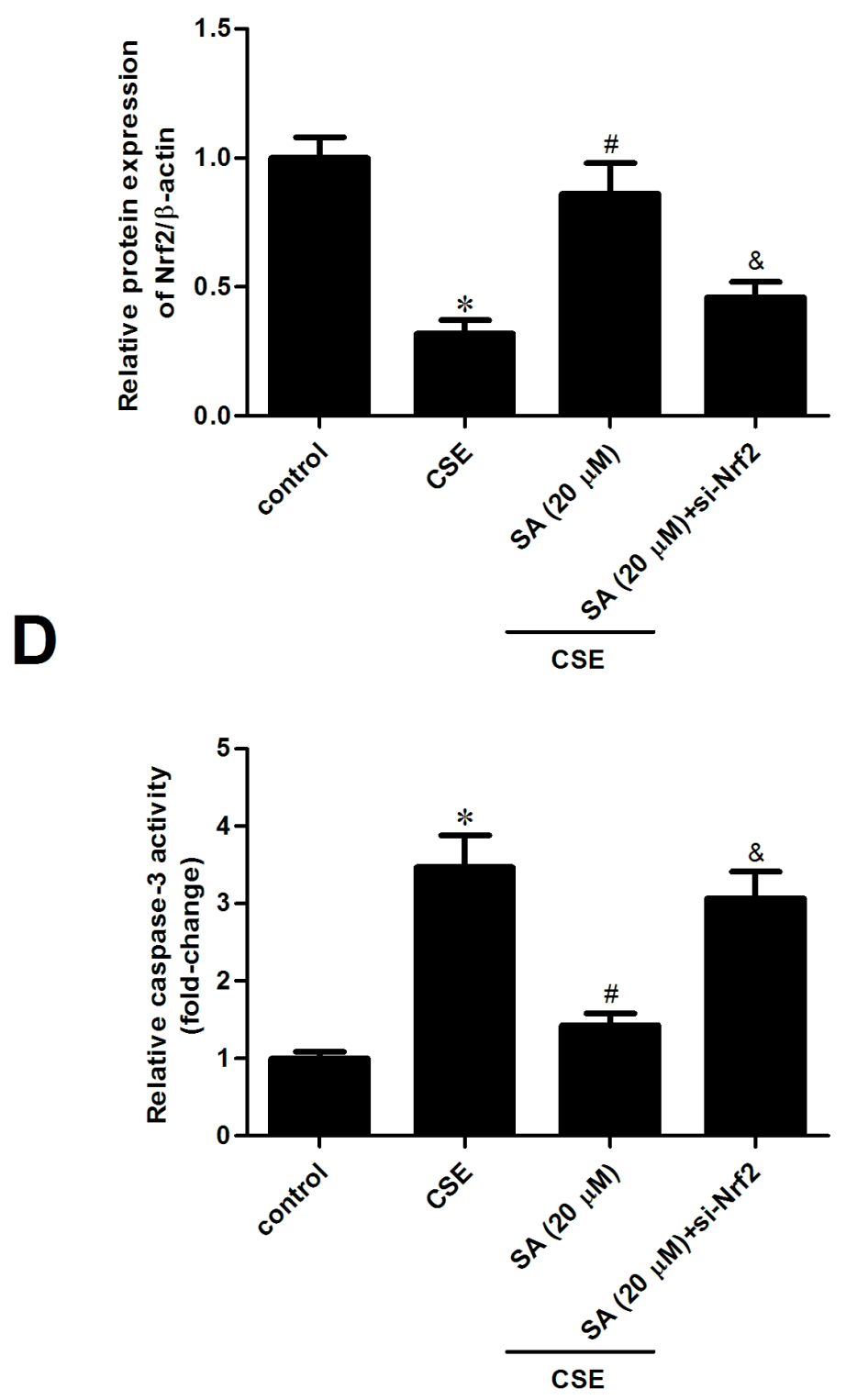

Figure 8

Knockdown of Nrf2 abrogated the protective effect of SA on CSE-stimulated 16HBE cells. 16HBE cells were transfected with si-Nrf2 for $24 \mathrm{~h}$ in the presence of $20 \mu \mathrm{M}$ SA before CSE stimulation. (A) The protein level of Nrf2 was detected by western blot analysis. (B) Quantitative analysis of the relative protein level of Nrf2. (C) The result of ROS production in 16HBE cells. (D) Caspase-3 activity was detected. ${ }^{*}<0.05$ compared to control group; $\# p<0.05$ compared to CSE group; \&p<0.05 compared to CSE+SA group. 